$\underline{\text { Supporting Information (SI) }}$

\title{
Direct Synthesis of Porous Polyurea Films by Vapor Deposition Polymerization in Ionic Liquid
}

Yuya Ohsawa, Rikuto Takahashi, Shingo Maruyama and Yuji Matsumoto*

Department of Applied Chemistry, School of Engineering, Tohoku University, 6-6-07, Aramaki Aza Aoba Aobaku, Sendai, Miyagi 980-8579, Japan

*E-mail: matsumoto@atomol.che.tohoku.ac.jp

The supporting Information includes one scheme, one Table and four Figures. 


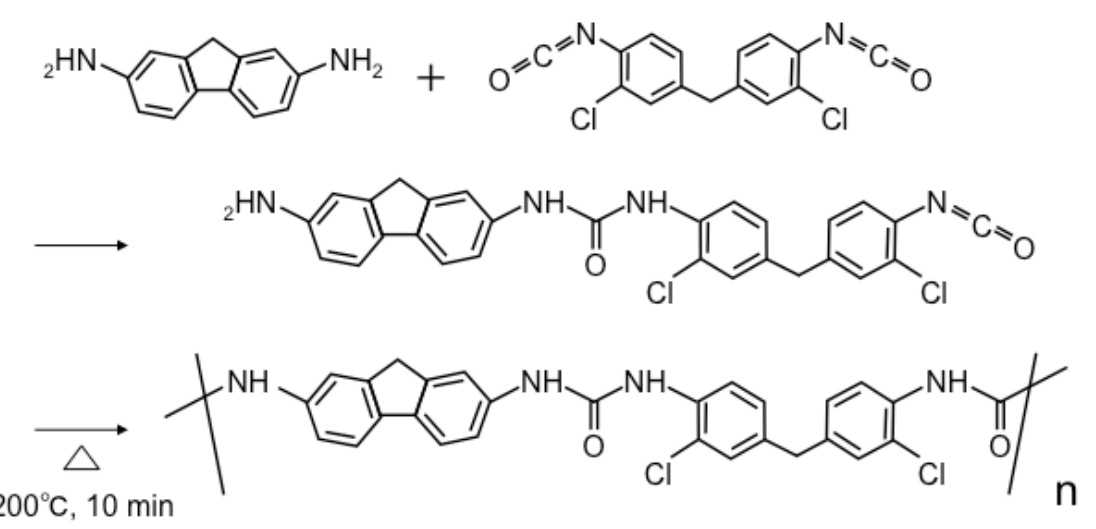

Scheme S1: Reaction of DAF and MBCI into PU. 


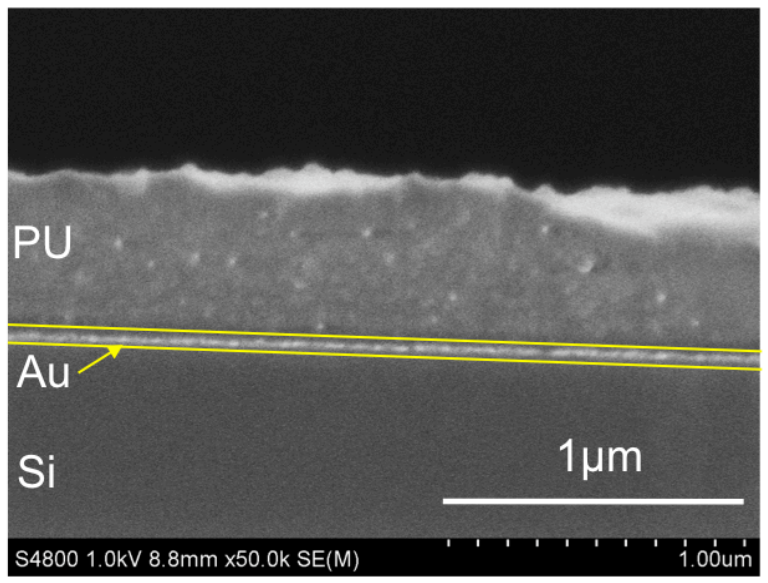

Figure S1: A cross-sectional SEM image of the porous film, whose thickness was determined to be about $530 \mathrm{~nm}$, thicker than the nominal thickness of $360 \mathrm{~nm}$ due to the porosification, giving a volume fraction of voids to be 0.32 . 


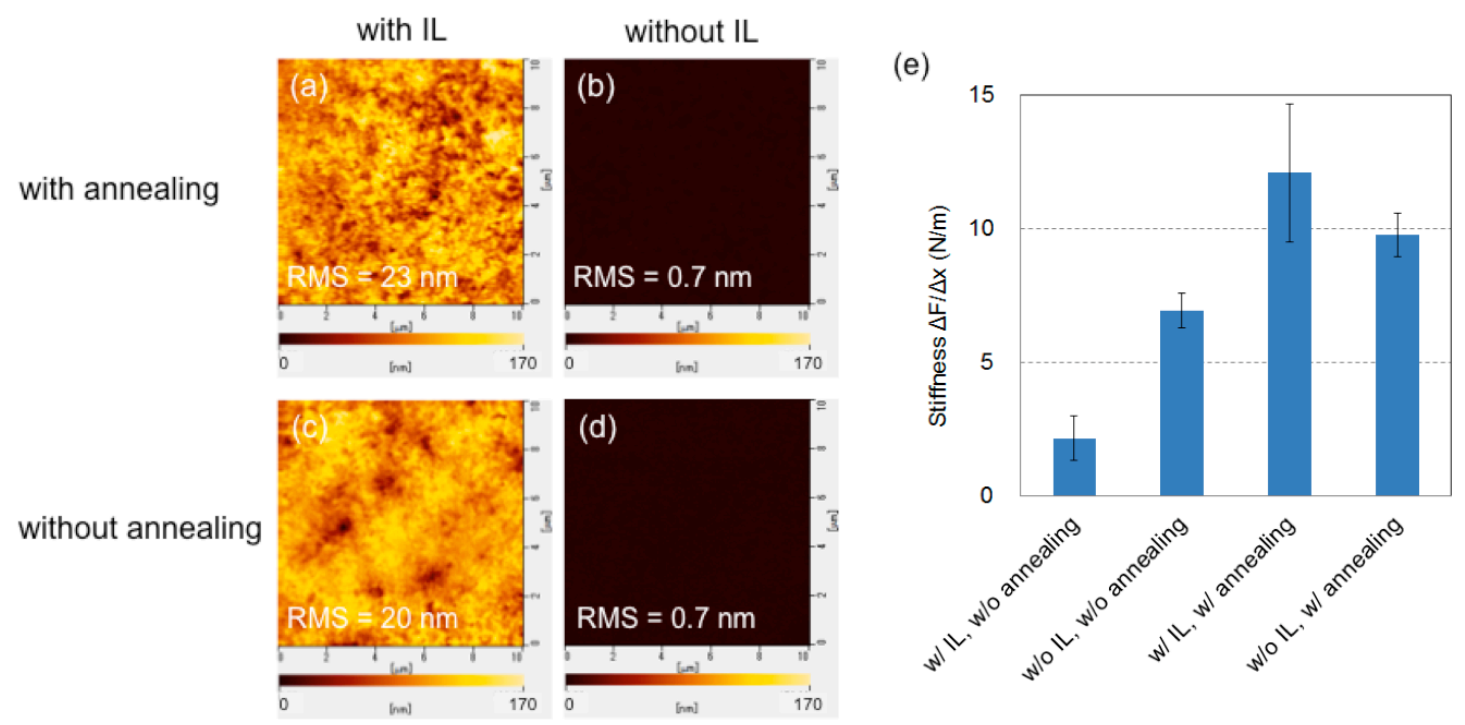

Figure S2: The average values of stiffness $\Delta F / \Delta X(N / m)$ for each polyurea film, calculated from AFM force curves taken at 10 points randomly selected from the scan area $(10 \mu \mathrm{m} \times 10 \mu \mathrm{m})$ are shown (e), together with the corresponding AFM images (a)-(d). 
(a)

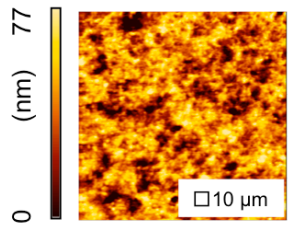

(b)

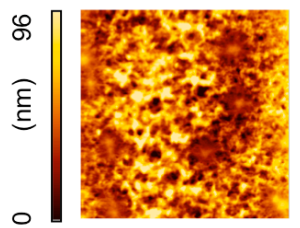

(c)
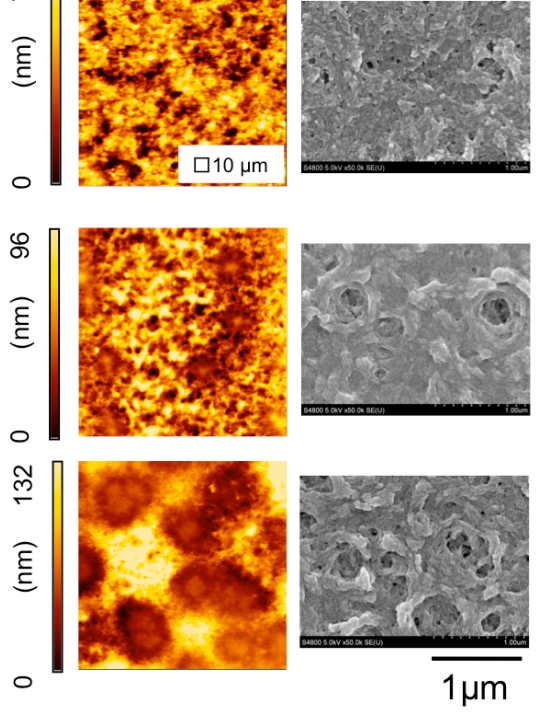

(d)

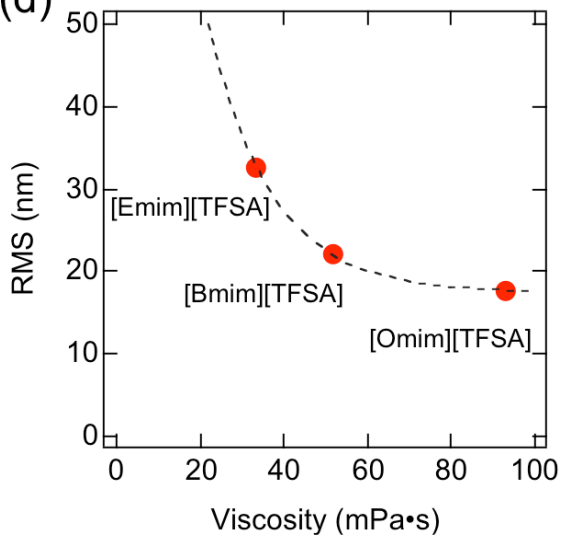

Figure S3: Network structures of polyurea films fabricated with three ILs with different viscosities of (a) [Omim][TFSA], (b) [Bmim][TFSA] and (c) [Emim][TFSA]. (d) The pore size (RMS roughness value) increases with a decrease of the viscosity of the IL. 


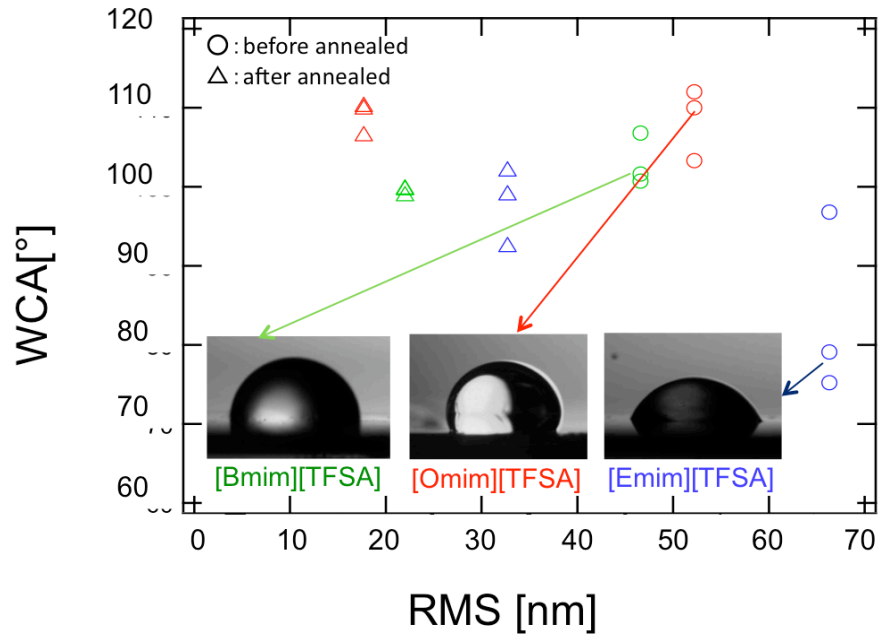

Figure S4: The plots of the CAs against the RMS values for various PU films prepared with different ILs.

Table S1

\begin{tabular}{|c|c|c|}
\hline \multirow{2}{*}{ Ionic liquid } & \multicolumn{2}{|c|}{ WCA [degree] } \\
\cline { 2 - 3 } & After annealed & Before annealed \\
\hline$[$ Omim] $[$ TFSA $]$ & 108.8 & 108.5 \\
\hline$[$ Bmim] $][$ TFSA $]$ & 99.4 & 103.0 \\
\hline$[$ Emim] $][$ TFSA $]$ & 97.8 & 83.7 \\
\hline
\end{tabular}

